\section{Movement detection thresholds and stimulus duration*}

\author{
RONALD L. COHEN \\ Department of Behavioral Science, University of Toronto, Toronto 5, Canada \\ and \\ CLAUDE BONNET \\ Sorbonne-Universite de Parist, Paris, France
}

Movement detection thresholds were measured for varying exposures of a moving spot. A tradeoff was found in which an increase in duration ( $T$ ) was offset by a decrease in the velocity required for detection (V). In the range of durations studied (about 50-700 msec), $V \times T$ was constant. The $V \times T$ constancy was interpreted in terms of the direct detection of movement as motion, and a comparison was made with Bloch's law.

The threshold for the detection of movement has been measured in many studies (Graham, 1965). However, few studies have systematically examined the relationship between the rate of movement at threshold and the duration for which the movement is exposed. Most studies that have addressed themselves to the investigation of the effect of duration (T) on threshold velocity (V) have tended to use a relatively wide range of exposure durations. Dimmick and Karl (1930) investigated the range $0.5-4.0$ sec, Brown and Conklin (1954), the range $0.5-16.0 \mathrm{sec}$, and Leibowitz (1955a), the range 0.125-16.0 sec. All three studies reported that increasing the duration of exposure lowered the threshold velocity. In no case was a one-to-one tradeoff between $V$ and $T$ reported, although examination of the data of both Dimmick and Karl (1930) and Leibowitz (1955a) suggests that such a tradeoff may occur with relatively short durations.

A more recent study, one by Henderson (1971), investigated the relationship among the variables associated with motion detection, including exposure duration. The emphasis in this study was on the tradeoff between the duration $(T)$ and the extent (S) of the movement at threshold for relatively high rates of motion (greater than 10-20 min/sec) and extremely short exposures (miniexposures, less than the order of 50-100 msec). Data were also obtained for exposures up to about $1 \mathrm{sec}$, also suggesting the possibility of a tradeoff between $V$ and $T$ in the range 100-1,000 msec.

\footnotetext{
*This research was conducted at the Sorbonne, while Dr. Cohen was visiting on a grant from the Swedish Council for Social Science Research. He was, at that time, on staff at the University of Uppsala, Sweden. + Laboratoire de psychologie Expérimentule et Comparée associé au Centre National de la Recherche Scientifique, Paris.
}

The following experiment was designed to study the relationship between $V$ and $T$ over a range of what may be termed short exposures (as distinct from miniexposures), in order to test the prediction that there is, in fact, a one-to-one tradeoff between $V$ and $T$, with $V \times T$ constant at threshold.

\section{METHOD}

Movement thresholds were measured following two procedures: (1) Constant duration procedure (Tc), where thresholds were measured for constant durations by varying the velocity and, consequently, the length of the track of motion; and (2) constant velocity procedure (Vc), where movement thresholds were measured for constant velocities by varying exposure duration and, consequently, the length of the track.

\section{PROCEDURE}

The $S$ sat in a semidark room with his head in a headrest. At a horizontal distance of $3.4 \mathrm{~m}$ from his eyes was a translucent screen, $75 \times 50 \mathrm{~cm}$, having a luminance of $0.10 \mathrm{~mL}$. Onto this screen could be projected, from behind, a small light spot, $6 \mathrm{~mm}$ in diam and of $0.40 \mathrm{~mL}$ luminance, which moved clockwise in a circular path, $12 \mathrm{~cm}$ in diam. Coincident with the point at which the moving spot appeared was a removable fixation spot, $8 \mathrm{~mm}$ in diam, $19 \mathrm{~cm}$ from the top of the screen and centrally placed in the horizontal plane.

The moving spot could be lighted and extinguished by means of an electronic timer system, so that the duration and length of the motion path could be varied. In the experiment, the spot was exposed only at the top of the circular path, and, regardless of the length of arc exposed, the starting point always remained the same and very close to its zenith.

When using the constant duration procedure, an exposure time was selected. The velocity of the motion could be varied continuously in the range $20-0.8 \mathrm{~min}$ of visual angle/ $\mathrm{sec}$, and absolute movement threshold measurements were made using descending series of velocities only (i.e., from movement to no movement responses). Durations used were 50 , $70,90,120,150$, and 200 msec. Each $S$ came on three separate occasions, and different random orderings of the duration were used in each session.

The $S$ fixated the fixation spot. The $E$ stood behind the screen and could see when the moving lamp, which provided the light spot, began to approach the onset end of its arc, at which time $E$ warned $S$ by saying, "Ready." The fixation spot, which was faint enough to prevent any visible afterimage, was extinguished manually by $E$ about $0.5 \mathrm{sec}$ before the onset of the lamp. The $S$ was to continue fixating that part of the screen where the fixation spot had been until the moving spot had disappeared at the offset end of its arc, at which point he was to report movement or no movement. Instructions to the Ss were to try to respond to motion rather than to displacement. The velocity of the spot was decreased on successive trials until S reported no movement on two consecutive trials.

Fixation was not controlled during the actual threshold measurements. However, only experienced Ss were used and, to test fixation under experimental conditions, trials were run prior to the experiment, using an electrode eye-movement recorder. These tests showed that an $S$ was quite capable of maintaining fixation, even after disappearance of the fixation spot, so it is assumed that the thresholds were measured under conditions of at least approximate fixation.

With the constant velocity procedure, the velocities tested were $1.14,2.29,4.01,5.44,8.59$, and $11.27 \mathrm{~min}$ of visual angle/sec. Thresholds were measured by varying the duration in steps of $10 \mathrm{msec}$. The Ve procedure was similar to that used with the Tc procedure, and, again, only descending series were used. On no trial was the exposure so short that the moving spot was not perceived by the $S$.

The same three $\mathrm{Ss}$ were used for both conditions. Each $\mathbf{S}$ came on six occasions and received each condition three times. The order of testing of conditions was randomized over the six sessions.

\section{RESULTS}

Table 1 gives the mean values of $V$ using the $T c$ procedure and of $T$ using the Vc procedure. Also given are the between-Ss standard deviations.

Plots of $\log V$ against $\log \mathrm{T}$ (means) 
Table 1

Mean $V$ alases of Exposure (T) and Velocity (V) for Detection of Movement (Expressed in $M \sec$ and $M$ in of Visual Angle Per Sec, Respectively)

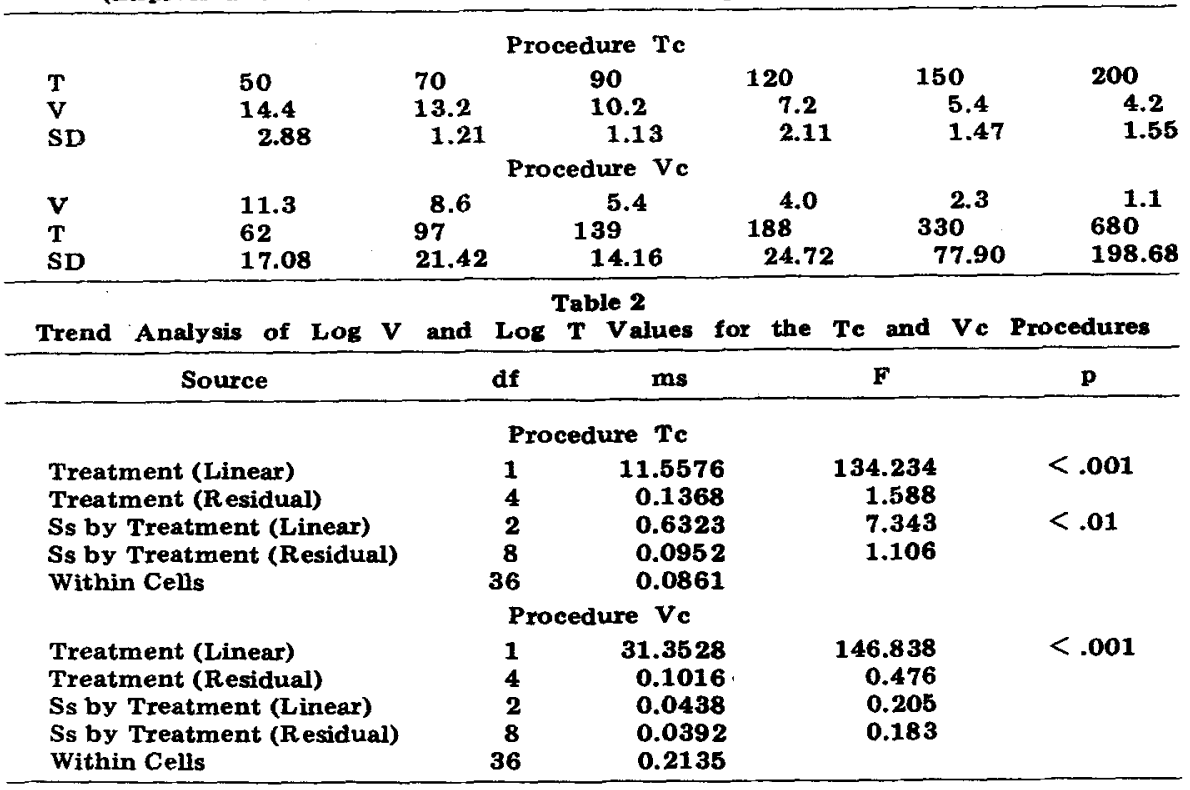

are shown in Fig. 1. Two trend analyses were carried out, using the log values (six treatment levels, three $\mathrm{Ss}$, three observations per cell). It was necessary to use the transformed values in the analyses, at least in the case of the Ve data, because of the variation of the $S D$ with the mean. Linear coefficients for the unequally spaced values of the independent variables were calculated according to the method given in Draper and Smith (1966, p. 156). Table 2 gives the results of these analyses.

Using the within-cells variance as error term gave a significant linear component but no significant residual component (quadratic, cubic, quartic, and quintic) for both sets of data. For procedure Tc, the significant $S_{s}$ by Treatment (linear) interaction means that there were slope differences among Ss. However, the common linear component is still very close to the .05 level of significance when $\mathrm{S}$ by Treatments (linear) is used as error term, $F(1,2)=18.279$.

The regression equations for the best fitting linear plots are given in Fig. 1. The slopes of these plots can be regarded as -1.00 , for both procedures, which means that $V \times T$ at threshold is constant.

In spite of the remarkably close correspondence between the data and the prediction that $\mathrm{V} \times \mathrm{T}$ is constant, the between-Ss differences would indicate caution in generalizing from these results. However, analysis of Leibowitz's (1955a) data shows V x T values at threshold for durations of $0.125,0.25,1.00$, and $2.00 \mathrm{sec}$, averaged over the luminance range the constancy did not even extend as far as the 2 -sec exposure duration.

The data in Henderson's (1971) study are presented graphically, which makes it difficult to abstract precise figures. However, from the curves, it does appear as if $S$ (i.e., $V \times T$ ) is constant as $V$ varies in the range of 1 to $10-20 \mathrm{~min} / \mathrm{sec}$.

Considering the findings of the present study in the light of these earlier studies greatly increases the probability that the relationship $\mathrm{V} \times \mathrm{T}$ $=$ a constant at threshold has general application, at least for durations in the range 50 msec to $1-2$ sec.

The data reported by Brown and Conklin (1954) do not show a V x T constancy, but this may be due to the method of measurement, which produced very much higher threshold values than the other three studies discussed above. It also should be noted that most of the measurements made in the latter study involved exposures of $1 \mathrm{sec}$ or more.

\section{DISCUSSION}

$0.16-5.0 \mathrm{~mL}$, to be $2.19,1.73,2.05$, and $2.18 \mathrm{~min}$ of visual angle, respectively. This shows a fairly high degree of constancy. Similar constancy is shown in the Dimmick and Karl (1930) data for the two lowest durations $(0.5$ and $1.0 \mathrm{sec})$, which give $V \times T$ values of 1.28 and $1.19 \mathrm{~min}$ of visual angle. In the Leibowitz (1955a) study, the constancy shown for the lower exposure durations was not apparent with the 16-sec exposure; in the Dimmick and Karl (1930) study,
The constancy of the $V \times T$ values can be interpreted in two ways: (1) The spot must move through a minimum displacement $(0.7-0.9 \mathrm{~min}$ of visual angle in the present experiment) for movement to be detection rather than with motion detection, and (2) although a constant displacement appears to be necessary for the detection of movement, we are, in fact, dealing with motion detected. The implication is that we are dealing with displacement

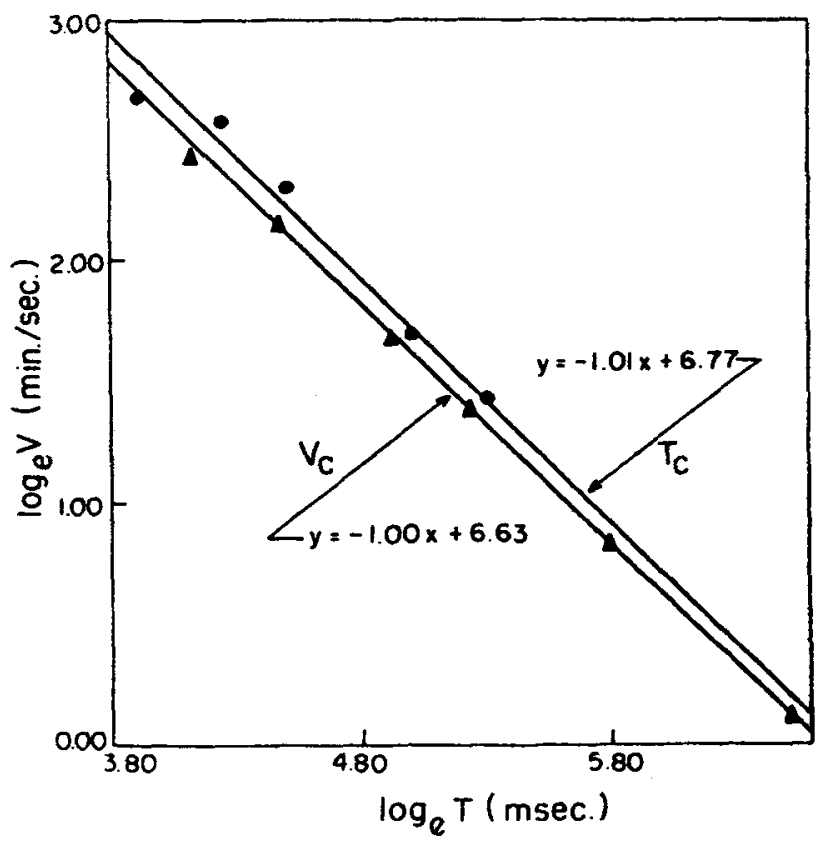

Fig. 1. Plots of $\log \mathrm{V}$ against $\log \mathrm{T}$ (means) for the Tc and Ve procedures. 
detection rather than with displacement detection.

The problem of two kinds of movement threshold is, of course, an old issue. It has long been accepted that movement can be detected either directly, as motion, or else indirectly, by inference from displacement (Exner, 1885; Brown \& Conklin. 1954; Brown, 1955; Cohen, 1964; Graham, 1965; Bonnet, 1971). However, there has been no general agreement as to which conditions favor which kind of detection.

Dimmick and Karl (1930) favor a complete explanation of movement detection in terms of displacement thresholds. They propose that for short exposures (less than $1 \mathrm{sec}$ ) the displacement of the movement must exceed that of the "spatial limen" for movement, to be detectable; for long exposures, they suggest that movement thresholds are related to the "spatial limen for successive stimuli."

Leibowitz (1955b) supports a two-process model, where movement is detected directly as motion at relatively short exposures but is implied from displacement at long exposures. Evidence cited in favor of this distinction is that the presence of reference lines in the field of movement drastically lowers the threshold for perceived movement at long exposures (16 sec) but does not affect the threshold at short exposures $(0.25 \mathrm{sec})$. Leibowitz (1955a) also notes that the general shape of the functions relating luminance and threshold velocity are similar to those relating luminance and intensity discrimination, flicker, grating acuity, and vernier acuity. He concludes that motion thresholds may depend on similar mechanisms to those underlying the other types of discrimination, and that, for short exposures, photochemical mechanisms are critical for all the above types of discrimination, including motion detection.

Accepting Leibowitz's distinction between displacement and motion thresholds in principle, it is proposed that the conditions of the present experiment were conducive to the detection of motion rather than displacement. Further, since $V \times T$ appears to be constant within a critical range of exposure durations, it is proposed that the rate of the motion should be regarded in the same way as is brightness in luminance detection thresholds, namely, as an intensity measure. Bloch's law states that, within certain limits, intensity $x$ exposure duration is constant at the luminance detection threshold. Similarly, for motion detection, the intensity of the motion, that is its rate, $x$ exposure duration, is also a constant. (This interpretation makes the assumption that Ss did not vary their criteria for detecting movement with varying exposure times in the above experiment. While it is possible that such a criterion change did occur, the obtained V $\mathrm{V}$ T constancy does suggest that a constant criterion was used.)

The analogy between Bloch's law and the $\mathrm{V} \times \mathrm{T}$ constancy obtained for motion thresholds breaks down in that Bloch's law holds up to only about 100 msec (Bartlett, 1965), whereas the constancy relationship between velocity and duration appears to hold up to about 1-2 sec. However, whereas photochemical mechanisms provide the theoretical basis for Bloch's law, such mechanisms are not being proposed for motion detection. Physiological studies with animals have produced evidence for the presence in the visual system of detectors that are especially sensitive to motion (see, for example, Barlow \& Hill, 1963a, b). There is also evidence for such detectors at the human level (Sekuler \& Ganz, 1963; Cohen, 1965; Pantle \& Sekuler, 1969; Bonnet \& Pouthas, 1972). Since the perception of motion is supposedly related to the rate of firing of motion-sensitive detector cells (Barlow \& Hill, 1963a, b) and not to photochemical processes, the difference in the range of applicability for Bloch's law and the $V \times T$ constancy relationship is not a critical issue.

It would also appear that the $V \times T$ constancy relationship breaks down at the lower end of the scale, that is, for miniexposures. For miniexposures, Henderson (1971) reports that, at threshold, the extent of the movement varies inversely with the duration, which he interprets along the same lines as Dimmick and Karl (1930). Perceived motion, and, in Henderson's case, more especially perceived direction of motion, is the result of the retina's being able to discriminate successive events. He argues against an energy explanation in favor of an interaction model where extent and duration are considered to be basic variables interacting to give motion perception.

It is also possible to explain Henderson's (1971) data for miniexposures in terms of motion detectors. The rate of firing of motion detectors in the rabbit, for example, has been shown to be dependent on the rate of the motion (Barlow \& Hill, $1963 a, b)$. The $V \times T$ constancy found in the present experiment is consistent with the notion that the detection of motion involves a critical number of firings, rather than a critical rate of firing, corresponding to $V \times T$. If it is simply assumed that there is a limit to the rate at which motion detectors can fire, then increases in $\mathrm{V}$ will be unable to of fset decreases in $T$ above a certain value of $\mathrm{V}$, say about $10-20 \mathrm{~min} / \mathrm{sec}$. The only way in which the number of firings can be increased to the critical value under miniexposure conditions is by increasing the number of receptors involved, that is, by increasing the extent of the motion. Thus, in the range investigated here, the critical number of firings is attained by a tradeoff between $V$ and $T$. However, for very fast movement, increasing $V$ can no longer offset a decrease in $T$, for which reason an increase in $S$ also becomes necessary. For long exposures, above 1-2 sec, the authors agree with Leibowitz $(1955 \mathrm{a}, \mathrm{b})$ that the detection of displacement becomes increasingly important. The implication for the motion detector model is that with slow velocities, the time between firings from successive detectors is too great for the required integration of firings from several detectors to occur. That is, there is a limit on the time allowed for the detector system to attain the critical number of firings necessary for the direct detection of motion.

\section{REFERENCES}

BARLOW, H. B., \& HILL, R. M. Selective sensitivity to direction of movement in ganglion cells of the rabbit retina. Science, 1963a, 139, 412-414.

BARLOW, H. B., \& HILL, R. M. Evidence for a physiological explanation of the waterfall phenomenon and figure after-effects. Nature, I963b, 200 , 1345-1347.

BARTLETT, N. R. Thresholds as dependent on some energy relations and characteristics of the subject. In C. $H$. Graham (Ed.), Vision and visual perception. New Yodk: Wiley, 1965.

BONNET, C. Les mécanismes de la perception de la vitesse d'un mouvement visuel. Bulletin de Psychalogie, 1971, 24. 415-417.

BONNET, C., \& POUTHAS, V. Interactions between spatial and kinetic dimensions in movement aftereffects. Perception \& Psychophysics, 1972, 12 (2B), 193-200.

BROWN, $R$, H, Velocity discrimination and the intensity-time relation. Journal of the Optical Society of America, 1955, 45 189-192.

BROWN, R. H. \& CONKLIN, J. E. The lower threshold of visible movement as a function of exposure time. American Journal of Psychology, 1954, 67. 104-110.

COHEN, R. L. Problems in motion perception. Uppsala: Lundequistska Bokhandel, ig64.

COFEN, R. L. Adaptation effects and after-effects of moving patterns viewed in the periphery of the visual field Scandinavian Joumal of Psychology. $1965,6,257-264$.

DIMMICK, R. L \& KARL, J.C. The effect of the expoesure time upon the R.I. of visible motion. Joumal of Experimental Psy chology, 1930, 13, 365-369.

DRAPER, N. R., \& SMITH, H. Applied regression analysis. New York: Wiley. 1966.

EXNER, S. Ein Versuch über die Netzhautperiphene als organ zur Wahmehmung von Bewegungen. Pfiügers Archiv, 1885, 38, 217-218.

GRAHAM, C. H. Perception of movement. 
In C. H. Graham (Ed.), Vision and visual perception. New York: Wiley, 1965.

HENDERSON, D. C. The relationships among time, distance, and intensity as determinants of motion discrimination. Perception \& Psychophysics, 1971, 10, 313-320.

LEIBOWITZ, H. W. The relation between the rate threshold for the perception of movement and luminance for various durations of exposure. Journal of Experimental Psychology, 1955a, 49, 209-214.

LEIBOWITZ, H. W. Effect of reference lines on the discrimination of movement. Journal of the Optical Society of America, $1955 \mathrm{~b}, 45,829-830$.

PANTLE, A., \& SEKULER, R. Contrast response of human visual mechanisms sensitive to orientation and direction of motion. Vision Research, 1969, 9, $397-406$.

SEKULER, R. W., \& GANZ, L. After-effect of seen motion with a stabilized retinal image. Science, 1963, 139, 419-420.

(Accepted for publication June 6, 1972.) 